RONGRONG FU, Ph.D. ${ }^{1}$

(Corresponding author)

E-mail: frr1102@aliyun.com

MENGMENG HAN, Master Candidate ${ }^{1}$

E-mail: hanmm1008@foxmail.com

BAO YU, Master Candidate ${ }^{1}$

E-mail: yb1_517@stumail.ysu.edu.cn

PEIMING SHI, Ph.D. ${ }^{1}$

E-mail address: spm@ysu.edu.cn

JIANGTAO WEN, Ph.D. ${ }^{1}$

E-mail: wens2002@163.com

${ }^{1}$ Measurement Technology and Instrumentation

Key Lab of Hebei Province, Yanshan University

No. 438 Hebei Street, Qinhuangdao 066004, China
Human-Transport Interaction Original Scientific Paper Submitted: 27 June 2019 Accepted: 19 Feb. 2020

\title{
PHASE FLUCTUATION ANALYSIS IN FUNCTIONAL BRAIN NETWORKS OF SCALING EEG FOR DRIVER FATIGUE DETECTION
}

\begin{abstract}
The characterization of complex patterns arising from electroencephalogram (EEG) is an important problem with significant applications in identifying different mental states. Based on the operational EEG of drivers, a method is proposed to characterize and distinguish different EEG patterns. The EEG measurements from seven professional taxi drivers were collected under different states. The phase characterization method was used to calculate the instantaneous phase from the EEG measurements. Then, the optimization of drivers' EEG was realized through performing common spatial pattern analysis. The structures and scaling components of the brain networks from optimized EEG measurements are sensitive to the EEG patterns. The effectiveness of the method is demonstrated, and its applicability is articulated.
\end{abstract}

\section{KEY WORDS}

electroencephalogram (EEG); weighted brain networks; driver fatigue;

\section{INTRODUCTION}

Electroencephalogram (EEG) is capable of direct measurement of brain activity [1-3]. The information from EEG is one of the most predictive and reliable indicators for evaluating human cognition and the study of human state discrimination $[4,5]$. Unfortunately, EEG recordings are generated from the cortex and collected from the scalp. In EEG measurements there always appear complex and non-linear characteristics [6-10]. Understanding the non-linear and complex dynamics underlying EEG measurements is a significant and challenging problem [11]. In this regard, a primary task is to characterize and quantify different EEG patterns without the influence of the non-linear and complex dynamics underlying EEG. Generally speaking, a common practice is to examine the phase fluctuation properties containing all relevant or discriminatory information needed to solve the state discrimination problem. Driving is a cognitively effortful task requiring a mental capacity of organizing and processing a great deal of information simultaneously [12-14]. The driver's state is reported as one of the principal factors in driving safety [15]. For taxi drivers or long-haul bus drivers, fatigue reduces their ability to operate vehicles safely and reduces their situation alertness [16]. Therefore, characterizing EEG patterns to infer brain intentions and brain states is important and has wide applications.

The purpose of this paper is to introduce an instantaneous phase-based method to characterize typical patterns from experimental EEG measurements. Our idea is that the phase fluctuations associated with time series are caused by the intrinsic dynamics and can therefore yield important information about the underlying differences that the existing, non-phase-based methods are incapable of revealing. In particular, given a set of experimental EEG measurements, we first use the empirical mode decomposition (EMD) method pioneered by Huang et al. [17] to extract the phase fluctuations. To uncover any robust scaling behaviour hidden in the phase fluctuations, the scaling analysis by brain networks of EEG 
measurements is used. Our main finding is that the relationship between the structure and the function is an integration problem. And for each of two distinct patterns arising typically in the driving state EEG measurements, a scaling exponent can be extrapolated from the phase fluctuations. For different EEG patterns, the degree distributions are distinct, indicating the effectiveness of the combined EMD and functional brain network method to characterize and distinguish complex EEG patterns.

\section{EXPERIMENTS AND DATA}

\section{ACQUISITION FOR DRIVERS' EEG MEASUREMENTS}

Seven professional taxi drivers (male, ages 3040) were recruited on their voluntary basis in this study. The time of the experiment was settled at approximately 3 p.m. The taxi drivers should have already worked for more than 5 hours during that experiment day. The participants were asked to stop driving to cooperate with the EEG signal collection. After the experiment, they received a certain compensation for participating. The drivers admitted that they were in fatigue condition after long-time driving, so the first recorded data which lasted for three minutes should be labelled as fatigue, then ten minutes were given to the drivers to rest. "Rest" means the driver is in the taxi with his eyes closed. After relaxing another three minutes the data were recorded, which is regarded as post-relax. The experiment protocol is given in Figure 1 .

The Emotiv EEG recorder was used for recording these EEGs in both fatigue and post-relax situations. The location of electrodes in the International 10-20 System of Emotiv is shown in Figure 1. The channels used were AF3, F7, F3, FC5, P7, O1, O2, P8, FC6, F4, F8, and AF4. The sample rate was $128 \mathrm{~Hz}$.

\section{PHASE CHARACTERIZATION OF EEG MEASUREMENTS}

To see the phase characterization of EEG measurements, analytic signal $y(t)$ can be constructed from the EEG data sequence $x(t)$ by the Hilbert transform,

$$
y(t)=P\left[\frac{1}{\pi} \int_{-\infty}^{+\infty} \frac{x\left(t^{\prime}\right)}{\left(t-t^{\prime}\right)} d t^{\prime}\right]=A(t) e^{i \varphi(t)}
$$

where $P$ indicates the Cauchy principal value for integral; $A(t)$ represents the instantaneous amplitude, and $\varphi(t)$ represents the instantaneous phase.

Figure 2 shows trajectories of the analytic signal in its complex plane for a signal and the extracted intrinsic modes from the EEG measurement. As

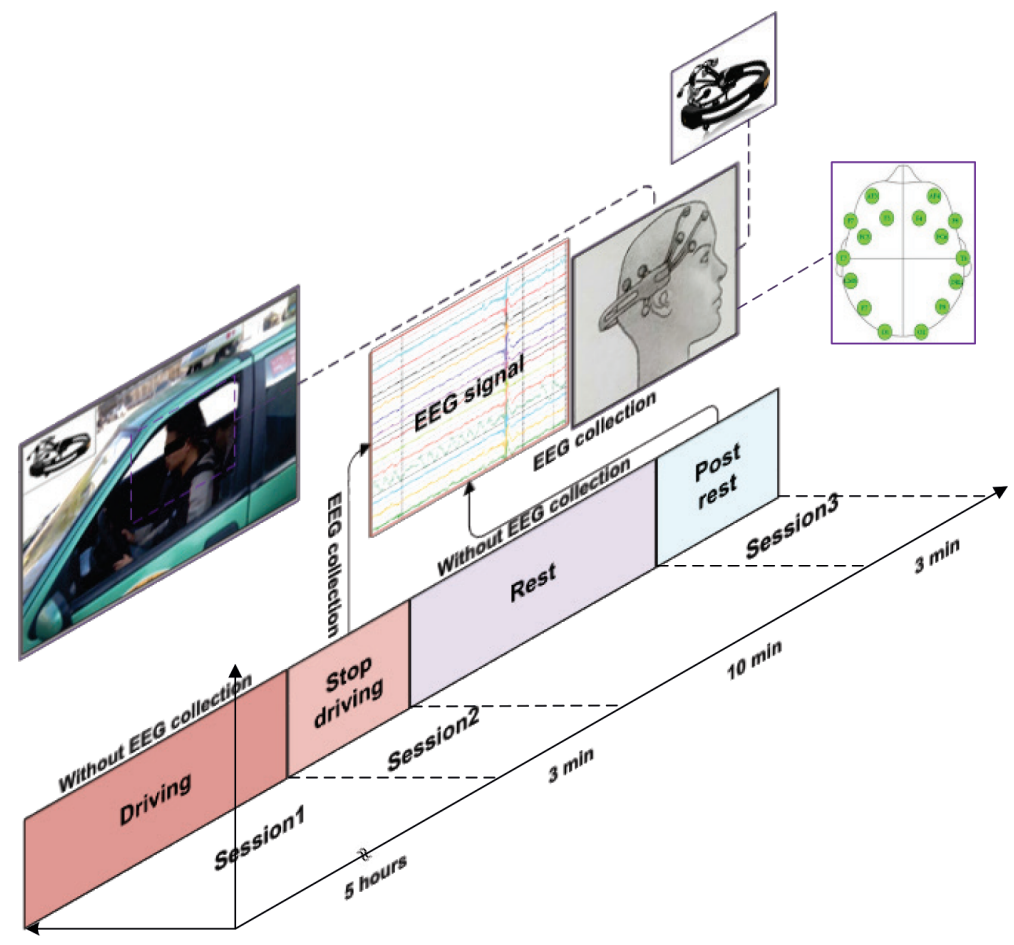

Figure 1 - Setup of experiment and study protocol 
shown in Figure 2a, due to multiple centres of rotation in the original signal, a properly defined phase variable cannot be obtained from the analytic signal.

A proper phase can be defined based on the following two conditions in its complex plane $[18,19]$ : (1) there is a preferred direction of rotation; and (2) the rotation can be defined with respect to a unique centre. The final proper phase can be obtained from the complex conjugate pair.

However, the signal collected in real condition is always too complicated to satisfy these two conditions. Thus, it is necessary to decompose the original signal into a number of modes whose analytic signals correspond to proper rotations with the empirical mode decomposition (EMD) method. EMD method can decompose signal $x(t)$ into a finite sum of Intrinsic Mode Function (IMF), which is to identify the innate undulations belonging to different time scales and sift them out to obtain one intrinsic mode at a time $[16,17]$. When the sifting process is stopped, the signal is shown as,

$$
x(t)=\sum_{j=1}^{n} c_{j}(t)+r_{n}(t)
$$

where the intrinsic modes $c_{j}(t)$ are nearly orthogonal to each other, and residue $r_{n}(t)$ is a monotonic function. By using the Hilbert transformation, each mode $c_{j}(t)$ generates a proper rotation in the complex plane of its analytic signal,
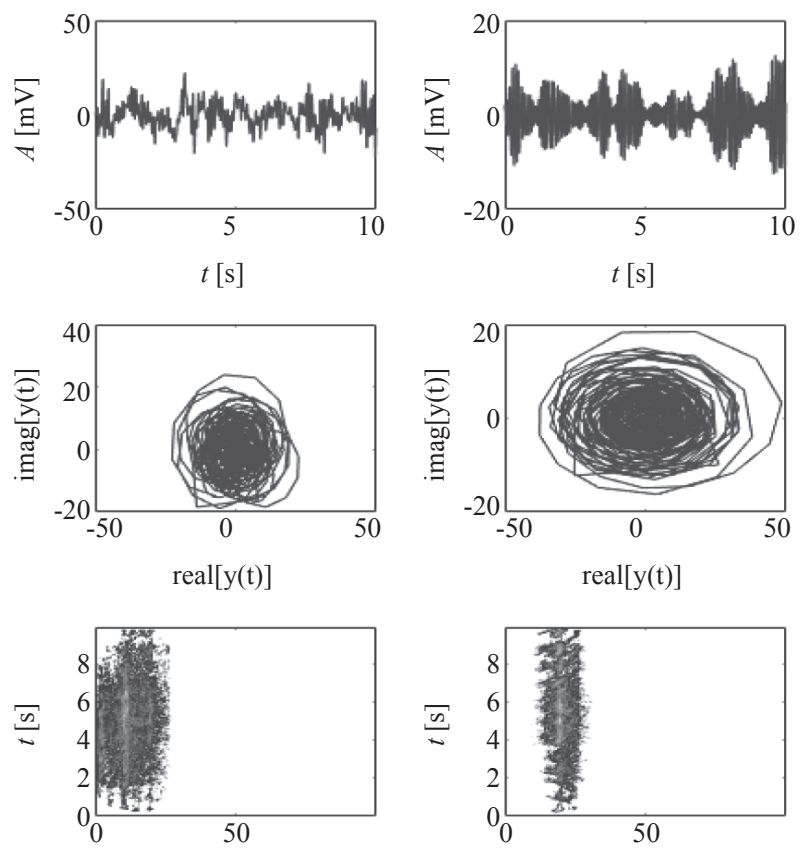

$f[\mathrm{~Hz}]$

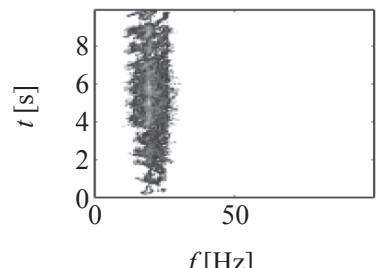

$c_{j}(t)=A_{j}(t) e^{i \varphi_{j}(t)}$

where $A_{j}(t)$ represents the $j$-th amplitude function and $\varphi_{j}(t)$ represents the $j$-th phase function. They can be obtained from the analytic signal of the $j$-th intrinsic mode function. And the expected rotation frequencies can be written as,

$\omega_{j}(t)=<\frac{d \varphi_{j}(t)}{d t}>$

The phase function of different intrinsic mode functions and time-frequency domain description for EEG measurements, has been analysed. The results can be seen in Figure 2. In this three-by-four figure matrix, the first row shows time-domain description of the original signal and its principal IMFs. The second row shows the original signal and its IMFs Trajectory in the complex plane. The third row of this figure matrix gives the frequency-time distribution for the corresponding signals in the first column. The fact is obtained that although the original EEG measurements are complex, its phase consists of only a small number of non-trivial proper rotations.

The frequency distribution of the second IMF is within the proper frequency range of beta band (13$30 \mathrm{~Hz}$ ) of EEG signal; the third IMF is of $8-13 \mathrm{~Hz}$ within the frequency range of alpha-band; the other IMFs with lower and narrower frequency distribution
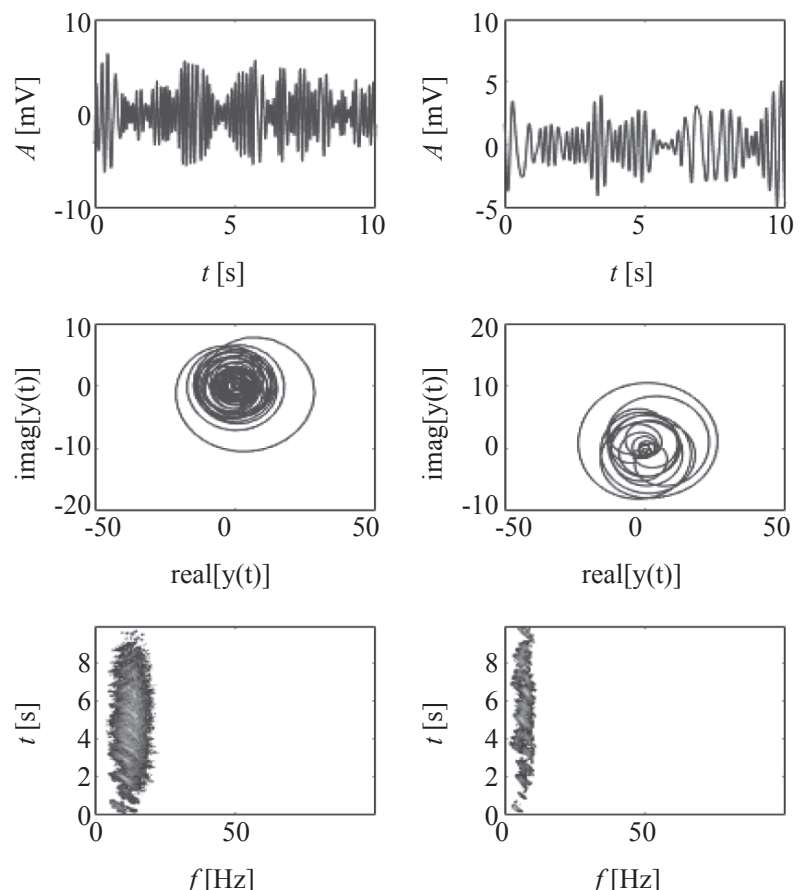

Figure 2 - Time-domain description, trajectory in the complex plane of the analytic signal from EEG, and frequency-time distribution for the original signal and its principal IMFS 
contain low-frequency noise reasoning from breath and body movement. Therefore, the third IMF is chosen as alpha band for the following analysis.

\section{SCALING ANALYSIS BY BRAIN NETWORKS FOR DRIVING TASKS}

The adjacent matrix is obtained by computing the magnitude squared coherence between signals from two different channels $[20,21]$. The magnitude squared coherence $C_{x y}$ is a function of the power spectral densities $P_{x x}(f)$ and $P_{y y}(f)$ of $x$ and $y$ $[7,22]$, and the cross power spectral density $P_{x y}(f)$ of $x$ and $y$,

$$
C_{x y}(f)=\frac{\left|P_{x y}(f)\right|^{2}}{P_{x x}(f) P_{y y}(f)}
$$

Therefore, by using Equation 5, the adjacent matrix can be computed, as shown in Figure 3. The brain networks under different states have different structural information as the connectivity between different pairs of nodes [22]. In order to give a flavour about the distribution of spatial patterns, Figure 3 provides the weighted functional brain network, in which the spatial topographic distribution obtained a noticeable difference to driver's different states. The fluctuations associated with different driver states can be reflected in the network organization, as shown in Figure 3. The subfigures in the upper line of Figures $3 a-3 d$ are configured by EEG measurements from the states after long-time driving, and the driver can feel fatigued clearly in this regard. And the subfigures in the lower line of Figures $3 e-3 h$ are configured by EEG measurements from the states after rest, and the driver fatigue is relieved a lot in this state. From Figure 3, the distinct driver states can be reflected in weighted brain networks (Figures $3 b$ and $3 f$ ) and node degree distributions (Figures $3 c$ and $3 g$ ). When the driver fatigue relieves after rest, the weighted brain network is composed of a set of highly connected nodes, and this can be considered as an atlas of connectivity. The brain network under fatigue state has a structure with lower connectivity between different pairs of nodes. The node degree distributions are used to quantify the structural connectivity quantification between two distinct driver states.

The degree distribution $P(k)$ of a network is defined to be the fraction of nodes in the network with degree $k$ [23-25]. If there are $N$ nodes in total in a network and $n_{k}$ of them have degree $k$, the degree distribution can be given by:
$P(k)=\frac{n_{k}}{N}$

The two subfigures in the last column of Figures $3 d$ and $3 h$ show that the node degree with the highest occurrence rate is seven for driver state after longtime driving, and the node degree with the highest occurrence rate is eleven for driver state after rest, which is marked with "**" in Figure 3. The nodes degree distribution increase during the fatigue level variation, the probability distribution of nodes degree moves from smaller degree values to larger degree values. Because the nodes degree represents the number of connectivity belonging to the nodes, the connectivity of the whole weighted brain network increases when the driver recovered from fatigue by some time rest.

\section{DISCUSSION}

The scaling analysis results are shown here for other participants of the driving tasks by functional brain network to explore the consistence among other participants. From the adjacent matrix to the weighted brain networks, the analysis results of the driving task for the other six participants can be illustrated in Figure 4. For most of these six participants, the connectivity of brain networks increases as the driver fatigue relieves after rest. The brain network under fatigue state has a structure with lower connectivity between different pairs of nodes. The node degree distributions are used to quantify the structural connectivity quantification between two distinct driver states, as illustrated in Figure 5. From Figure 5, the distinct driver states can be reflected in node degree distributions clearly.

The subfigures with horizontal bars in Figure 5 show the node degree with the highest occurrence rate marked with "*". In this six-by-four figure matrix, subfigures in each row are for the same driver, the subfigures in the first two columns are for the driver state just after long-time driving, the last two columns represent the node degree distributions for the state after rest. During this fatigue level variation, the nodes degree distribution increases for most participants; the probability distribution of nodes degree moves from smaller degree values to larger degree values as illustrated in Figure 5. Because the nodes degree represents the number of connectivity belonging to the nodes, the connectivity of the whole weighted brain network increases when the driver recovers from fatigue by some time rest. 


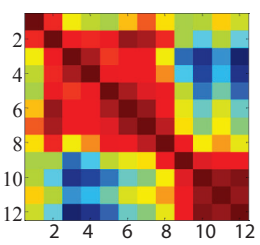

a)

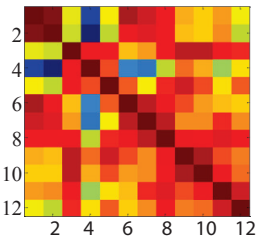

e)

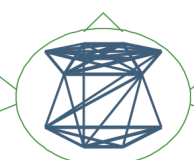

b)

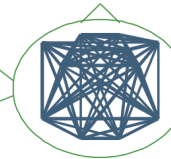

f)

State after long time driving

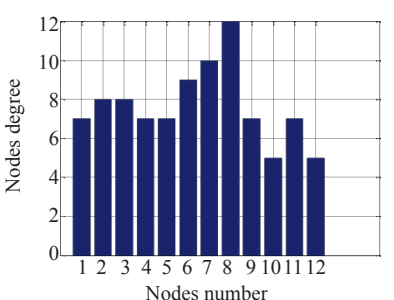

c)

State after rest

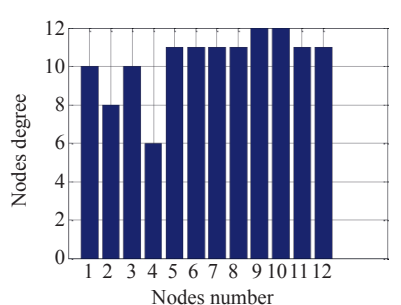

g)

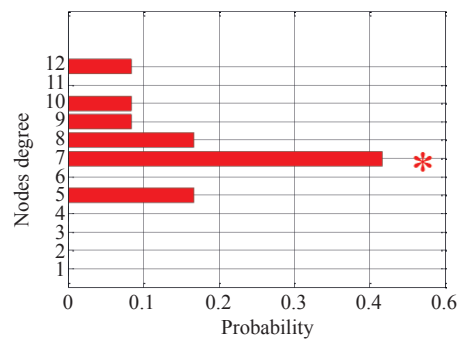

d)

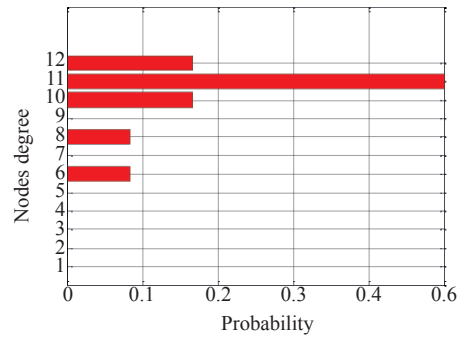

h)

Figure 3 - The weighted brain networks and degree distributions for driver states: a), e) adjacent matrix; b), f) weighted brain network; $c$ ), g) bar plot of nodes degree; d), h) bar plot of probability of nodes degree

State after long-time driving
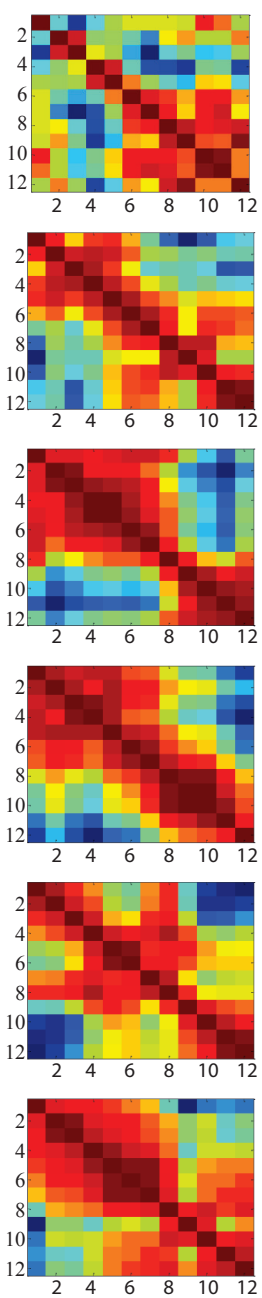
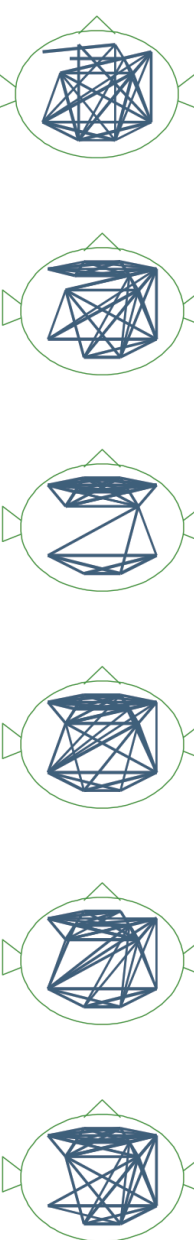

State after rest
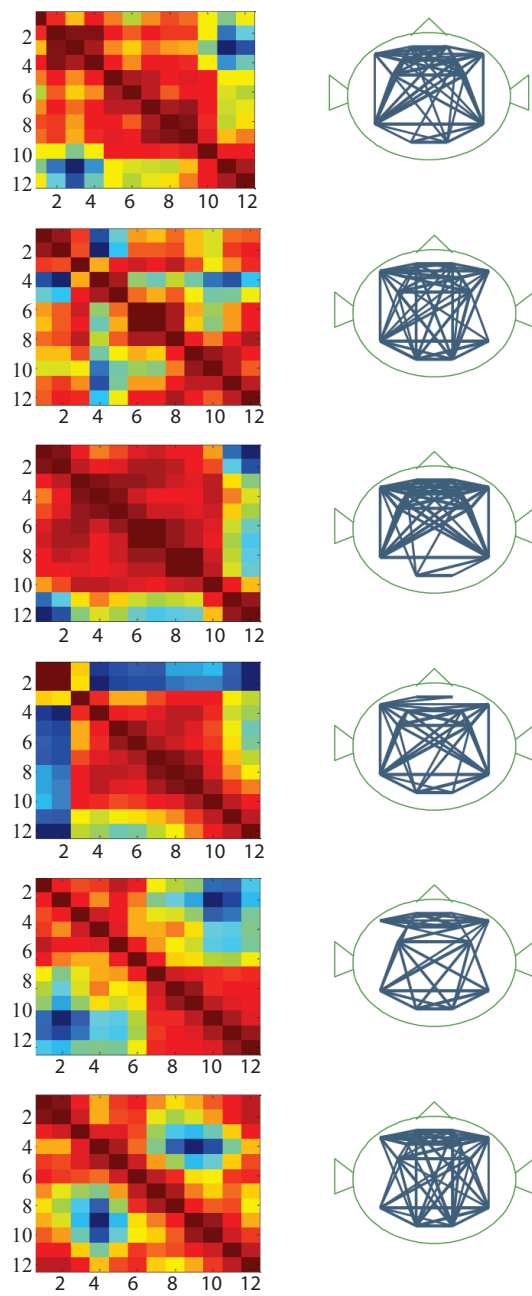

Figure 4 - Weighted brain network of six drivers during driving state variations 

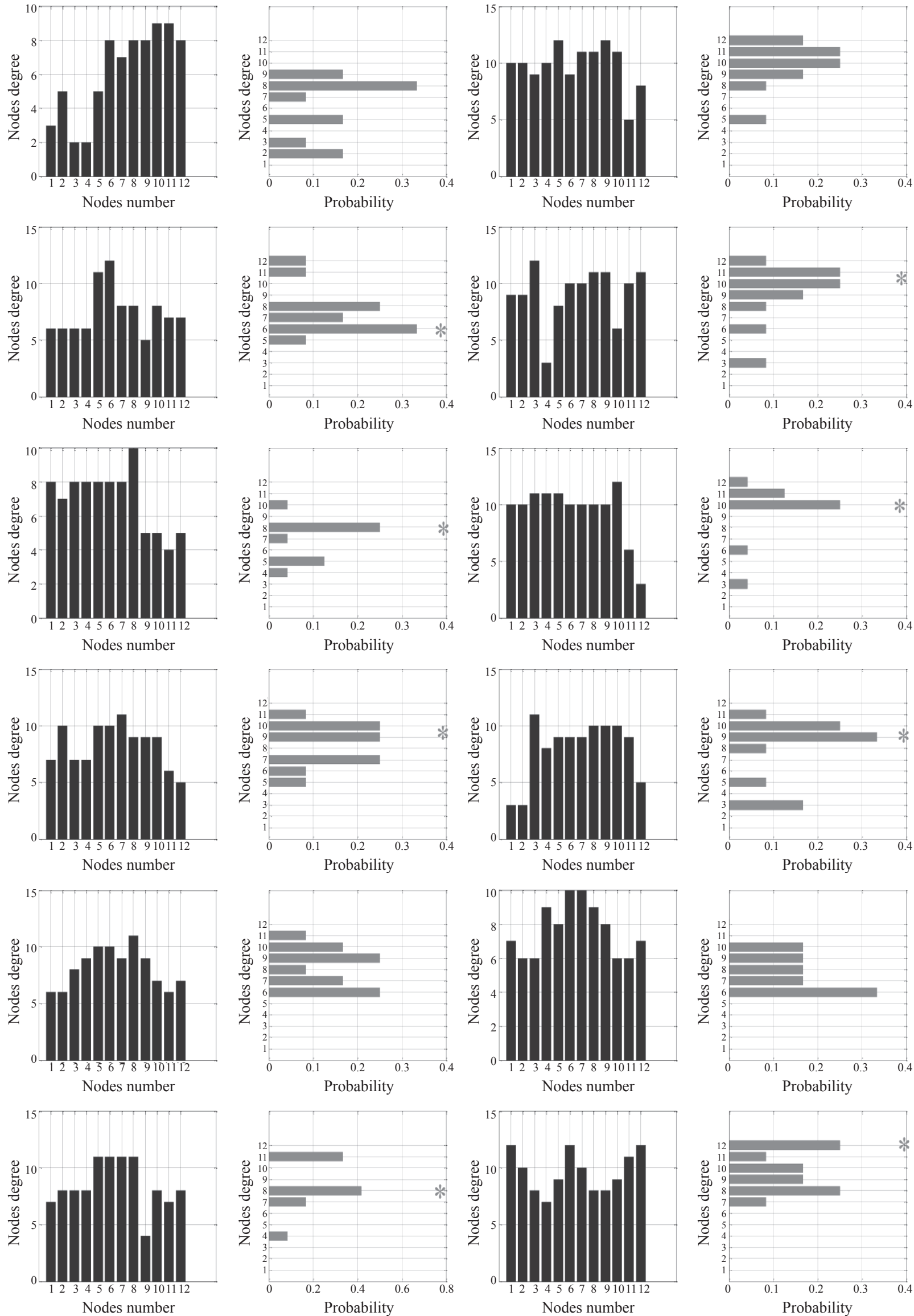

Figure 5 - Nodes degree distribution of six drivers during driving state 
The variation of degree distribution can be obtained from Figure 6 for all these seven drivers by bar plot of the average value of node degrees with an error bar. This variation trend can be found in most drivers, except drivers E and F; they may have had not enough rest for this experiment. However, the average degree value increases for the other drivers. This means that the number of functional connectivity increases as well.

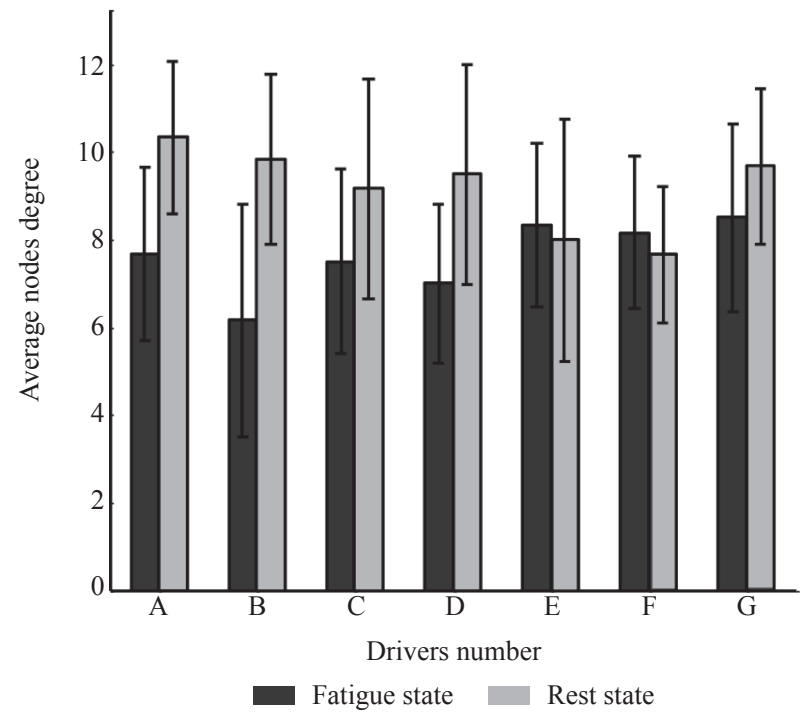

Figure 6-Nodes degree distribution of seven drivers during driving state variation

The current study explored the whole-brain functional connectivity of the driving fatigue tasks based on an instantaneous phase-based method. Our idea was inspired by previous studies [15, 26], which suggests that the phase fluctuations of non-linear signals such as EEG measurements are sensitive to changes in human cognition behaviours. These phase fluctuations associated with time series are caused by the intrinsic dynamics and can therefore yield important information about the underlying information that the existing, non-phase-based methods are incapable of revealing. These are validated by the discriminant of different states in human cognition-related tasks: driving fatigue.

To uncover any robust behaviour hidden in the phase fluctuations, the weighted brain networks of EEG measurements processed by EMD were used. EMD as a processing method is widely used in reducing artefact involving EEG measurements. Distinguishing from the conventional EMD-based analysis, the novelty of this work is that the EMD method is used as an instantaneous phase-based method to obtain robust behaviour of phase fluctuations for classifying distinct EEG patterns. The other novelty of this work lies in the fact that the graph theory was employed to gain more understanding of the reorganization of brain networks under different cognitive states: mental fatigue/relax. The weighted brain networks show significant differences under different states among the participants in both cognitive tasks as discussed above.

In the driving task, the current finding of increased functional connectivity among the entire brain when the driver states varied from fatigue to resting, suggests when the driver is in the fatigue state, and the dysfunctional interactions among different brain regions that may lead to deficits in cognitive processes that rely on such connectivity. After prolonged driving, induced mental fatigue can impair the cognitive skills. This consistent phenomenon has been reported previously. Therefore, the relationship between structure and function is an integration problem. And for each of two distinct patterns arising typically in the driving state EEG measurements, a scaling exponent can be extrapolated from the phase fluctuations.

It was investigated further how the brain functional network topology property was associated with the cognition process as the driving fatigue. The brain network related to the driving tasks exhibited lower node degree distribution than this property after rest. We found that the node degree distribution of the best bands combination of the EEG had network property that was significantly higher during the mental fatigue state than the rest state. For different EEG patterns, the degree distributions are distinct, indicating the effectiveness of the combined EMD and functional brain network method to characterize and distinguish the complex EEG patterns.

\section{CONCLUSION}

The message from the current research on driving tasks is that the phase fluctuation information can help reveal the brain state involving the weighted brain network. Despite task changes in our experiments, the node distribution of different participants' brains can reveal distinct brain states. For the driver fatigue task, the nodes degree distribution increases for most participants, the probability distribution of node degree moves from smaller degree values to larger degree values when recovering from fatigue. Therefore, the brain network under fatigue has low node degree distribution. 
Fatigue-related neural specificity or dedifferentiation resulting from fatigue may lead to fatigue-related declines in the modularity of brain-wide network organization. Increased within-network connectivity may reflect recovering from fatigue. Because of its involvement in a wide range of functional networks underpinning different cognitive processes, further research is needed to understand the complex relations between changes in the network organization and performance and to determine whether the functions discussed in this paper extend more widely to other components of the brain.

\section{ACKNOWLEDGEMENTS}

This work was supported by the National Natural Science Foundation of China (Grant No. 51605419, 61973262), the Natural Science Foundation of Hebei Province (Grant No. E2018203433), the China Postdoctoral Science Foundation (Grant No. 2016M600193), the Hebei Province Funding Project for Returned Overseas Scholar (Grant No. CL201727).

付荣荣 ${ }^{1}$, 副教授, 博士 邮箱: frr1102@aliyun.com 韩萌萌 ${ }^{1}$, 硕士研究生 邮箱: hanmm1008@foxmail.com 于宝 ${ }^{1}$, 硕士研究生 邮箱: yb1_517@stumail.ysu.edu.cn 时培明 ${ }^{1}$, 教授, 博士 邮箱: spm@ysu.edu.cn 温江涛1, 教授, 博士 邮箱: wens2002@163.com 测试计量技术及仪器河北省重点实验室, 燕山大学, 秦皇岛河北大街438号，河北省，066004中国

\section{用于驾驶员疲劳检测的标度脑电功能脑网络相} 位波动分析

\section{摘要:}

脑电复杂模式的表征是识别在重要应用领域中 不同心理状态的重要问题。本文基于驾驶员脑电提 出了一种方法来表征和区分不同的脑电模式。采集 了七名职业出租车司机在不同状态下的脑电。利用 相位特征法计算脑电信号的瞬时相位，通过共空间 模式算法对驾驶员的脑电信号进行分析，实现脑电 信号的优化。由优化后的脑电得到的脑网络结构和 尺度分量对脑电模式敏感，证明了所提方法的有效 性，并阐明了其适用性。

\section{关键词:}

脑电; 加权脑网络; 驾驶员疲劳

\section{REFERENCES}

[1] Tabibi Z, Borzabadi H H, Stavrinos D, et al. Predicting aberrant driving behaviour: The role of executive function. Transportation research part F: Traffic Psychology and Behavior. 2015;34: 18-28.
[2] Wang L, Wang H, Jiang X. A New Method to Detect Driver Fatigue Based on EMG and ECG Collected by Portable Non-Contact Sensors. Promet - Traffic \&Transportation. 2017;29(5): 479-488.

[3] Chen J, Wang H, Hua C. Electroencephalography based fatigue detection using a novel feature fusion and extreme learning machine. Cognitive Systems Research. 2018;52(6): 715-728.

[4] Fu R, Wang S, Wang S. Real-time Alarm Monitoring System for Detecting Driver Fatigue in Wireless Areas. Promet - Traffic\&Transportation. 2017;29(2): 165-174.

[5] Zhang C, Cong F, Wang H. Driver fatigue analysis based on binary brain networks. Seventh International Conference on Information Science \& Technology. IEEE; 2017; 485-489.

[6] Chen J, Wang H, Hua C, et al. Graph analysis of functional brain network topology using minimum spanning tree in driver drowsiness. Cognitive Neurodynamics. 2018;12(6): 569-581.

[7] Zhao C, Zhao M, Yang Y, Gao J, Rao N, Lin P. The Reorganization of Human Brain Networks Modulated by Driving Mental Fatigue. IEEE Journal Biomedical Health Informatics. 2017;21(3): 743-755.

[8] Wang F, Xu Q, Fu R, et al. Study of driving skill level discrimination based on human physiological signal characteristics. RSC Advances. 2018;8(73): 42160-42169.

[9] Fu R, Tian Y, Bao T, et al. Improvement Motor Imagery EEG Classification based on Regularized Linear Discriminant Analysis. Journal of Medical System. 2019;43(6): 169.

[10] Chen J, Wang H, Hua C. Exploring the fatigue affecting electroencephalography based functional brain networks during real driving in young males. Neuropsychologia. 2019;129: 200-211.

[11] Liu Z, Sun J, Zhang Y, Rolfe P. Sleep staging from the EEG signal using multi-domain feature extraction. Biomedical Signal Processing \& Control. 2016;30: 86-97.

[12] Huang KC, Huang TY, Chuang CH, et al. An EEG-Based Fatigue Detection and Mitigation System. International Journal of Neural Systems. 2016;26(4): 1650018.

[13] Zhang C, Wang H, Wu MH. EEG-based expert system using complexity measures and probability density function control in alpha sub-band. Integrated Computer-Aided Engineering. 2013;4: 391-405.

[14] Wang F, Wang H, Fu R. Real-Time ECG-Based Detection of Fatigue Driving Using Sample Entropy. Entropy. 2018;20(3): 196.

[15] Lin CT, Chuang CH, Kerick S, et al. Mind-Wandering Tends to Occur under Low Perceptual Demands during Driving. Scientific Reports. 2016;6(1): 21353.

[16] Shi PM, An SJ, Li P, Han DY. Signal feature extraction based on cascaded multi-stable stochastic resonance denoising and EMD method. Measurement. 2016;90: 318328.

[17] Huang NE, Shen Z, Long SR, Wu MC, et al. the empirical mode decomposition and the Hilbert spectrum for nonlinear and non-stationary time series analysis. Proceedings of the Royal Society of London. Series A: Mathematical, Physical and Engineering Sciences. 1998;454: 903-995.

[18] Chen YF, Atal K, Xie SQ, Liu Q. A new multivariate 
empirical mode decomposition method for improving the performance of SSVEP-based brain computer inter-face. Journal of Neural Engineering. 2017;14(4): 046028.

[19] Fu R, Wang H, Han M, Han D. Scaling Analysis of Phase Fluctuations of Brain Networks in Dynamic Constrained Object Manipulation. International Journal of Neural System. 2020;30(2): 2050002.

[20] Horwitz B. The elusive concept of brain connectivity. Neuroimage. 2003;19(2): 466-470.

[21] Wang F, Zhang X, Fu R, Sun G. EEG characteristic analysis of coach bus drivers based on brain connectivity as revealed via a graph theoretical network. RSC Advances. 2018;8(52): 29745-29755.

[22] Zhang C, Cong F, Kujala T, Liu W, et al. Network Entropy for the Sequence Analysis of Functional Connectivity Graphs of the Brain. Entropy. 2018;20: 311.

[23] Gwin JT, Ferris DP. Beta- and gamma-range human lower limb corticomuscular coherence. Frontiers in Human Neuroscience. 2012;6(5): 00258.

[24] Barrat A, Barthlemy M, Vespignani A. Dynamical processes on complex networks. Boston: Cambridge University Press; 2008. p. 11-19.

[25] Rubinov M, Sporns O. Complex network measures of brain connectivity: Uses and interpretations. Neuroimage. 2010;52(3): 1059-1069.

[26] Gao ZK, Jin ND. A directed weighted complex network for characterizing chaotic dynamics from time series. Nonlinear Analysis: Real World Applications. 2012;13(2): 947-952. 